\title{
Special Topic: Resonance Spectroscopy and Spectrometry
}

\author{
Mei-Kun Fan ${ }^{1}$. Cheng-Zhi Huang ${ }^{2}$
}

Published online: 18 August 2021

(C) The Nonferrous Metals Society of China 2021

Resonance exists very commonly ranging from elementary particles to large celestial bodies, creating variety of new things and phenomena. As a typical forced vibration, resonance makes a physical system to absorb more energy from the surrounding environment at its natural vibration frequency, leading up to enhancement effect and bringing new opportunities to improve sensitivity in analytical chemistry. On the other side, light (or more broadly, electromagnetic wave) is by far one of the important probe with quantum effect for analytical chemists. Countless light-based sensing methods have been developed and implemented in various fields. With light, target molecules can be identified and characterized. With light, vivid visualization of tissues and organs in vivo is made possible. Also, with light, chemical species information from light-year-away-stars can be derived.

As one of the branches of resonance science and technology, optical resonance spectroscopy and spectrometry have made significant advances with the modern nanoscience and nanotechnology. Resonances in nanosized systems have laid the foundations of a lot of optical spectroscopic techniques and spectrometry such as nucleic magnetic resonance spectroscopy, resonance enhanced Raman spectrometry, resonance light scattering spectrometry, plasmon resonance spectrometry, resonance energy transfer spectrometry.

Regarding the dynamic analytical light scattering research, Journal of Analysis and Testing organized this special issue including six research articles to provide readers a glance of the researches carried out in this field.

Mei-Kun Fan

mkfan@swjtu.edu.cn

Cheng-Zhi Huang

chengzhi@swu.edu.cn

1 Faculty of Geosciences and Environmental Engineering, Southwest Jiaotong University, Chengdu 611756, China

2 College of Pharmaceutical Sciences, Southwest University, Chongqing 400715, China
Dr. Huang and co-workers from China Academy of Engineering Physics reported a study on the photolysis route of nano explosive with vibrational spectroscopy. $2,2^{\prime}, 4,4^{\prime}, 6,6^{\prime}$-Hexanitrostillbene (HNS) is an insensitive high-energy explosive. The current practice in the field is to develop nanosized HNS to improve its detonation performance. Thus, it is important to put efforts into the understanding of the photolysis process of the chemical in its nano-form. Various spectroscopic methods, including plasmon resonance enhanced Raman spectroscopy, were utilized to monitor the photolysis process. The photolysis route of nano HNS was mostly the same as its bulk counterpart. In addition, the possible mechanism of UV-Vis-induced visible color change of the nano-HNS was explored for the first time.

Dr. Zhan and co-workers from Southwest University reported the AuNPs/graphene hybrids-based enzyme-free immunoassay for respiratory syncytial virus detection based on surface plasmon resonance. An extremely sensitive and affordable plasmonic immunoassay for respiratory syncytial virus (RSV) detection with the naked eye was realized based on AuNPs/graphene sheets. It was found that the limit of detection was $1.7 \mathrm{fg} / \mathrm{mL}$, which was 100 times more sensitive than an AuNPs-based method. The ultrahigh sensitivity could be attributed to the large number of well-dispersed AuNPs deposited on the graphene surface.

Dr. Liu and co-workers from Chongqing Normal University reported a visual sensing method for ascorbic acid (AA) based on silver triangular nanoplates. By manipulate the redox reaction among silver nitrate and AA, newly formed $\mathrm{Ag}$ atoms can deposit on the surfaces of $\mathrm{Ag}$ TNPs. Subsequently, the morphology of Ag TNPs transforms from triangle to circle, resulting in a more than $160 \mathrm{~nm}$ blue shift of LSPR peak. The corresponding color of the solution converting from blue to yellow with the concentration of AA can be observed by naked eyes within $15 \mathrm{~min}$.

Dr. Wang and co-workers from Southwest Jiaotong University reported a quantitative SERS method for the determination of antibiotic norfloxacin in environmental water samples. Though SERS has been widely utilized for chemical 
identification, the analytical merits of the method is still in developing due to its poor reproducibility. By forming a free standing Ag NPs containing liquid membrane, the reproducibility of the SERS substrate was dramatically improved. They found that the percent RSD of the SERS substrate could be smaller than $10 \%$, while the regression coefficient $\mathrm{R}^{2}$ was 0.995 . Thus, the antibiotics in water samples could be quantitatively analyzed.

Dr. Ling and co-workers from Yunnan University developed a method for the surface plasmon resonance based colorimetric determination of chromium(III) ions in water based on monohydrogen phosphate induced aggregation of silver nanoprisms. The mechanism of sensing is straightforward. Monohydrogen phosphate at high temperature could induce the aggregation of silver nanoprisms. The presence of chromium(III) could prevent such a process. The LOD of the proposed method was only $3.6 \mathrm{nmol} / \mathrm{L}$.

Dr. Xiao and co-workers from East China University of Technology reported the sensitive discrimination of zinc and cadmium ions using fluorescent porous organic polymer. A fluorescent bipyridine based porous organic polymer (Bpy-POP) containing aggregation caused quenching units (anthracene) and metal ions recognition units (bipyridine) was synthesized through the Schiff base condensation reaction. Upon the presence of zinc and cadmium ions, the fluorescence from Bpy-POP was not only enhanced but also red shifted. Combining these two factors, the two metal ions can be quantitatively determined and discriminated.

This special issue only accounts for a very small collection of results achieved in the field of resonance spectroscopy and spectrometry. In addition, as can be seen in the issue, the sensing protocols varies from naked eye to advanced sensing instruments such as Raman CCD, and the targets of analysis can be ions, molecules, and even virus. We hope that the collection here could offer the readers a glimpse into this field, and more topics could start from here. To conclude, we appreciate the above-mentioned contributions in this special issue and would like to thank all the authors and reviewers for their efforts. Also, we are grateful for the assistance from the Editorial Office of Journal of Analysis and Testing.

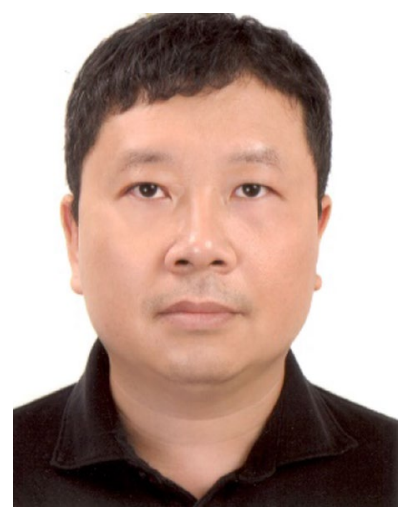

Dr. Mei-Kun Fan Professor in Environmental Analytical Chemistry, Southwest Jiaotong University. Dr. Fan obtained his PhD in Analytical/Physical Chemistry in 2010 from University of Victoria, Canada. After two short post-doctoral research experiences in University of Toronto and The Western University in Canada, he moved back to China in 2011 and became full professor of Southwest Jiaotong University in 2013. His research interest mainly lies in the applications of surface enhanced vibrational spectroscopy and localized surface plasmon resonance in environmental analytical chemistry. He also works on applications of nanomaterials in environmental remedy. He has more than 50 publications in the field, including 2 highly cited paper. He is also a member of the editorial board of the journal Applied Spectroscopy Reviews.

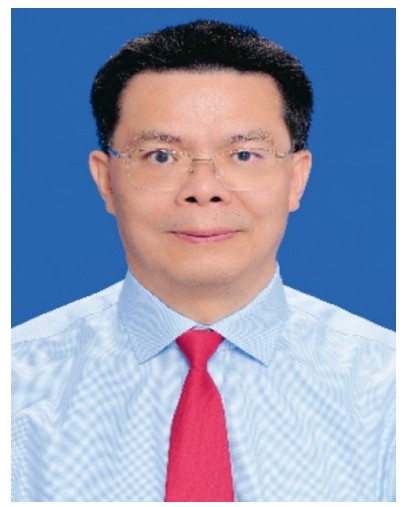

Dr. Cheng-Zhi Huang Professor of Analytical Chemistry and Pharmaceutical Analysis, Southwest University College of Pharmaceutical Sciences. After obtained Ph. D. in 1996 from Peking University, he became the research assistant or postdoctoral fellow in the Research Center of Hitachi Co. Ltd., Tokyo, Japan, Ottawa University, Ottawa, Canada, and the University of Tokyo, Tokyo, Japan. His research field is resonance spectroscopy and spectroscopy analysis, focusing on resonance light scattering spectroscopy, plasmonic resonance spectroscopy, and resonance energy transfer spectroscopy. He has published more than 500 research and review papers in academic journals including Angew. Chem. Int. Ed., ACS Nano, Chem. Sci., Green Chem., Biomaterials, Anal. Chem. Et al, and has published 1 research book, and 2 textbooks. 